\title{
Guest Editorial: Research, as Opposed to Dental Research - Oral Health Rather Than Dentistry
}

Given that guest editorials in the scientific literature provide rare occasions for the pontification and generation of controversy, such an opportunity is hard for me to resist. Nevertheless, to be contentious without being constructive is unproductive. That dentistry is facing a 'crisis' is generally agreed by all sectors of the profession. Clinicians see this in terms of 'busyness', academic administrators grieve over declining student quality or numbers and the closures or drastic re-organizations of schools. Faculty are demoralized and, of course, feel threatened by more stringent promotion-tenure requirements, just as the competition for research funding reaches an all-time plateau of difficulty.

What we are witnessing is a process that has been accelerating for some time, and which simply is an expression of the changing value system of society relative to public expenditure, specifically as it views the importance of dentistry as a health service.

The main purpose of this editorial is to raise awareness for the existence, causes, consequences, and gravity of such issues, and to provoke reactions that at least acknowledge that problems of our own creation may also be amenable to our own solutions. The title of this editorial is intended to encapsulate the essence of both the problem and the solution.

Contrary to our self-perception, we can conceivably be viewed by others as a profession that is to a major extent preoccupied with the technical aspects of preventing or correcting the consequences of two diseases, caries and periodontal disease, both microbial infections that (at least in theory), are preventable, but, in any case, are declining in prevalence. No matter how well-argued the case against such a perception, it is difficult to make a compelling justification for restoring the proportion of gross-national-product dollars, or fraction of health-related budgets that their maintenance would take, let alone provide growth in dentistry. The more imaginative of our colleagues point to newly discovered 'diseases' that, if incorporated in a new definition of dentistry, constitute 'unmet needs' that call for more and better treatment. Accordingly, treatment of such conditions as temporomandibular joint 'derangement' (both symptomatic and asymptomatic joint noises), or pain within a radius of six inches (i.e., $15.2 \mathrm{~mm}$. in metric units) of the dentition, or the newly popular obstructive sleep apnea, which, after all, involves the tongue (formerly a much neglected oral structure that previously only myofunctional therapists really 'treated'), are all among the emerging new subspecialties.

Many fanciful and biologically implausible treatment modalities have gained a wide measure of popular acceptance from practicing dentists. Since the advent of advertising and aggressive marketing of dentistry enjoined even by our professional organizations under the euphemism of public education campaigns, for which members are levied extra dues, the new face of dentistry has emerged as a high-tech profession with sophisticated tools, in search of problems. While magnetic resonance imaging is worth every cent if it can save my life, the investigation of my clicking joint may be entirely excessive or superfluous. This is particularly true if a gold standard does not exist for the identification of a disease, and especially in the absence of any evidence that the dentist who ordered this test is capable of interpreting the test results or of providing any treatment that has a predictable outcome with some known probability of success, whatever the criteria for assessment of this might be! How many clinicians understand that tests are only as useful as their sensitivity and specificity permit? How many of the diagnostic tests in dentistry have been evaluated for their accuracy of positive and negative prediction?

To be sure, all is not dismal in dentistry, and one can amass an impressive array of recent advances in dental technology that do represent innovations and are obviously enhanced methods for prevention or therapy. Composite restorations, bonded orthodontic brackets, advances in orthognathic surgery and post-surgical fixation, endosseous implants, and so forth, render possible what not so long ago was in the realm of the unimaginable. There are indeed grounds for justifiable pride in our achievements, even though there is little about which we can be complacent. Many opportunities still exist for further advances to be made. Providing that we become discriminating and differentiate between the fads and factually-supported problems, as well as their 'solutions', dentistry has an exciting future. This is precisely where research plays a role in determining the viability of the oral health field.

The problem is not one of stagnation, but rather one of resistance to change, coupled with a less-than-eager attitude to be critical of what we or our colleagues do, why we do it, how well we perform, and ultimately, the very question of how much good we do by accepting our traditional role in the grand scheme of health-care provision. In these days of superspecialization, where not all restorative dentists are gnathologists, where not all orthodontists treat craniofacial anomalies, but some proclaim the TMJ to be their 'field' and multirooted teeth require the services of specialist endodontists, where indeed there is a move for the creation of a specialty of implantology (if only it could be resolved whether it belongs to periodontics, prosthodontics, or oral surgery), few are the voices within our ranks that question if all of this is necessary, or good for either the public's health or for dentistry. Instead, the curricula of dental schools are finding niches to include an ever-widening spectrum of hot new stuff, while in many cases still clinging to 'requirements' for graduation that insist on more items of certain procedures and products than the demand level in the population can support. After all, when we graduated, we had to do umpteen surfaces of amalgam, still more of gold and more dentures than there were semesters; how then can today's graduates be competent unless they do as much? At least so goes the argument of some traditionalists to whom some deans feel that they must listen. Medical school graduates are not expected to be competent to pursue unsupervised independent practice immediately after graduation. Are dental graduates to be judged as competent merely on technical grounds, even if they may be unsure if a 'nucleotide' is a tidal wave produced by an atom bomb? My children in high school learn what a binomial distribution is and understand probabilities and decision analysis. How can health professionals interpret new developments unless they are taught as much as the lay public? How adaptable are such graduates to a lifetime of change, and how long is the half-life of their limited knowledge?

While all of this is going on, there also exists an unhealthy schism between the academicians and clinicians, both within and outside many of our dental schools. Administrations are challenging their deans to show just cause for retaining dentistry as an equal and viable component of the intellectual fabric of universities. This in turn requires at least lip service to the 'de rigeur' pursuit of scholarly activity, which implicitly threatens some clinical faculty with relegation to an apparently 
lesser-valued non-academic stature, or alternatively compels them to 'do' research. The natural resentment of those forced to engage in research without any particularly burning desire to answer any questions, nor the background necessary to enable them to formulate a testable question, turns not always on the system now forcing them to do so. The 'system' is too powerful, but the minority of those colleagues who establish the standard, who successfully compete in research with apparent ease, and obviously have the time to indulge in the esoteric and arcane because they do not work in the trenches and do not choose to be wet-fingered dentists, sometimes are seen as the root of the problem. When research workers question the very validity of treatment concepts or produce data that refute the clinical paradigm itself, then the gloves come off and the battle for credibility is on in earnest. The ubiquitous nature of this very human response to rapid change is 'dental school politics'. If only half the time and effort devoted to this pursuit were harnessed to productive ends, who knows how much further along we would be in terms of knowledge.

The discerning reader caught in the spell of this rhetoric may by now be asking why research rather than dental research and why oral health instead of dentistry? Admittedly, semantics may not lead to salvation, but a re-appraisal of philosophy and revision of objectives may point the way. During the years that I served on an NIDR Study Section, it was common for me to be berated by colleagues who were convinced that there was a conspiracy, in which I clearly participated or at least condoned, and which was directed at funnelling funds to basic scientists at the expense of 'dental research'. The 'dental research' that was supposedly so abused and stifled was generally clinical in thrust and therefore, according to the complainant, of more relevance to dentistry than basic science, even though it may admittedly have been plagued by those difficulties in design or scientific rigor that are so easy to fix for the testtube scientist! On more than one occasion, it was seriously suggested that there should be a special Study Section dedicated to clinical dental research that should be reviewed by real dentists who have a better appreciation for what clinical problems exist and for how difficult it is to do clinical dental research. 'Practicing' PhD's do not qualify as peers in this scheme. The fact that no such precedent exists in medicine failed to diminish enthusiasm for this suggestion.

Surely the solution is not that it be admitted ruefully that there are, or should be, different standards of science for dental research, as opposed to research in general. The paucity of science found in much of the dental literature and in the educational experiences to which clinicians are (regrettably, and through no fault of their own) exposed has been eloquently described in a recent editorial in this journal (Darvell, 1989). Clinical research is admittedly difficult to do well, but we not only have the innate ability to do this, but can also draw on the existing methodology developed for analogous problems in clinical medical research. There are methodological paradigms and established rules for assessment of the efficacy of treatments, the identification of risk factors, the cost-benefit and risk-benefit of clinical procedures, and so forth. Their application to dentistry is only just beginning, and still is questioned by some as a worthwhile scientific endeavor. It seems curious that for much of our history we have developed empirical treatments and clinical principles on nothing more than the collective conventional wisdom of clinicians, while being content to justify common practices 'post hoc', and all too often by indirect questionable inferences adapted from 'real' or 'basic' science. This state of affairs was in the past also a characteristic of medicine. However, their response to pressures for accountability, cost-effectiveness, and rationality have produced significant changes in priorities in education, re- search, and consequently, health care. Now it seems to be our turn to do likewise. This exhortation is definitely not an argument against the importance of fundamental investigation of biological processes. Bench research continues to be essential for unraveling the nature of mechanisms that underlie normal and abnormal processes. The plea is for the recognition of clinical science as an essential tool to direct teaching and the force to drive practice into a more enlightened, productive, and quite likely, a brighter future!

Finally, why oral health rather than dentistry? Our historical origins are derived from the Anglo-American tradition of the 'barber-surgeons' who also performed tooth pulling and evolved their art and craft to include replacing missing teeth with dentures, and later became more refined as they also incorporated the jewelers' skills. A parallel evolution occurred in other European nations, where medical schools recognized early that stomatology is a specialty of general medicine. Thus, the European dentist was a doctor of medicine who received specialty training in the management of oral conditions. Admittedly, the duration of such an educational program was insufficient to render its product as technically experienced or adept as those who served a longer apprenticeship in the dental arts and crafts. For this reason, the English-speaking world, where dentistry was/is viewed principally as the provision of appliances, regarded the European stomatologist-dentist as being inferior. For many reasons, it is perhaps time for us to consider whether our future role may not better be defined according to the stomatological model. 'Dentistry' has a definitely 'toothy' ring to it, and conjures the vision of the practitioner who is a "toothsmith' not unlike the blacksmith or plumber. Blacksmiths do not as a rule have university degrees in metallurgy-nor do they need one.

If we opt for oral health instead of dentistry, our academic disciplines, curricula, and departmental structures would have an altered focus, and clinical specialties may well be re-defined. The field of oral health includes, but is not necessarily confined to: (1) oral manifestations of local and systemic disease; (2) growth, development, and aging of craniofacial/oropharyngeal structures (anabolic and catabolic phases); (3) physiology of orofacial and pharyngeal function; (4) clinical epidemiology, technology assessment, and health-services research in oral health; and (5) therapeutic modalities in oral health maintenance. At present, our schools, and hence, our profession and its specialties, are predominantly organized according to the specifics and technical aspects that comprise therapeutic modalities. Given that technology is changing rapidly, that some of the technically demanding procedures are either delegatable to skilled ancillaries, or becoming easier to perform, and that a health service is ultimately based on using knowledge rather than on manual dexterity, can we limit ourselves to being dentists for much longer? Even if these suggestions are accepted as directions for the future, much remains to be done to bring about the necessary changes. Those in practice today will certainly not be made redundant in their lifetime. The question is whether the next generation of students and teachers of oral health are to be clones of the present or equal members of the medical community.

-- Peter S. Vig

Department of Orthodontics School of Dentistry The University of Michigan Ann Arbor, Michigan 48109-1078

\section{REFERENCE}

DARVELL, B.W. (1989): Guest Editorial: A Polemic on Behalf of a Poor Cousin, $J$ Dent Res 68:843. 